\title{
CHAOTIC ULTRASOUND GENERATION USING A NONLINEAR PIEZOELECTRIC MICROTRANSDUCER
}

\author{
Martial Defoort*, Libor Rufer, and Skandar Basrour \\ Univ. Grenoble Alpes, CNRS, Grenoble INP, TIMA, 38000 Grenoble, France
}

\begin{abstract}
We report on a piezoelectric micromachined ultrasonic transducer (PMUT) driven in a nonlinear regime, generating chaotic amplitude modulated ultrasonic waves. At large enough drives, the PMUT enters in the Duffing regime which opens a hysteresis with two available states. By modulating the frequency of the driving signal, the system may switch between both states, and selecting the appropriate modulation frequency enables to enter in the chaotic regime. The chaos is then imprinted as a modulation of the PMUT's amplitude. We characterize this regime in the three accessible domains: electrical, mechanical and acoustic, and demonstrate they are fully correlated. We then focus on the generated acoustic signals and demonstrate that the chaotic modulation propagates according to the PMUT's linear regime. Remarkably, the detected acoustic waves are strongly correlated to the on-chip piezoelectric measurements, regardless of the acoustic beam profile. The frequency spectrum of the chaotic modulation spreads around the ultrasonic career, mimicking a noise modulated career signal.

We exploit this property for jamming applications where the chaotic PMUT is used to mask surrounding acoustic waves. Unlike most jamming applications, our approach does not require driving signals with a broad frequency spectrum, the noisy pattern arising directly from the structure's dynamics. Using two PMUTs, one in the linear and the other in the nonlinear regime, we realize a proof-of-concept where the ultrasound generated by the first PMUT is drowned out by the chaotic PMUT signal. We demonstrate that the career frequency of the jamming PMUT does not need to match perfectly the one of the linear PMUT. This chaos generation is generic and could be adapted to any PMUT, and thanks to the rich frequency spectrum of the chaotic modulation, the frequency of the signal to jam does not need to be precisely known.
\end{abstract}

\section{KEYWORDS}

Microresonator, PiezoMEMS, PMUT, Nonlinear, Chaos, Ultrasound.

\section{INTRODUCTION}

Microelectromechanical Systems (MEMS) are versatile devices present in most recent technologies. They can be found as accelerometers [1], gyroscopes [2] microphones [3] or RF switches [4], with a broad range of geometries. Amongst these various MEMS applications, PMUTs enable to generate ultrasonic waves that are mainly used as position sensors through time-of-flight (TOF) measurements [5], for power transfer [6] or for wireless communication [7]. Because of their mechanical nature, MEMS are prone to present nonlinearities. Driven at large enough amplitude, these devices will present new properties enabling displacement amplification [8] or frequency stabilization [9]. These disruptive applications directly arise from the complex dynamics of the nonlinear process involved, reaching an apex with chaotic systems.

For a few decades, chaotic systems were found to present unique properties that the nonlinear physics community is still challenging [10]. The chaotic regime is at the boundary between stochastic and linear processes. On the one hand it is non-periodic, presenting a broad frequency spectrum and therefore sharing properties with white noise. On the other hand, it is a deterministic system, such that a full knowledge of initial conditions enables to completely predict the signal behavior. The richness of the chaotic regime arises from its sensitivity to initial conditions. In a real, physical chaotic system, noise (intrinsic or extrinsic) limits the full knowledge of its dynamics. This uncertainty exponentially increases with time, until it reaches the amplitude of the signal itself, losing all the information of the initial conditions. This property is at the essence of many applications in secured communication, especially for random number generators [11] and cryptography [12], but chaos has yet to reveal its full potential.

The generation of chaos in nonlinear MEMS has been a challenge for more than twenty years [13], with very few experimental realizations. By buckling a mechanical structure, the system enters in a bistable configuration, and if the device is driven with a large enough force and the appropriate frequency, it switches between the up and the down states with a chaotic pattern [13]. However, buckling a structure requires a large power, unconventional geometries, and / or specific materials, making the chaotic regime difficult to access and limiting its applications. Recently, a few works demonstrated a generic method to generate chaos, exploiting the Duffing regime of the nonlinear mechanical structures [14]-[16]. Through this technique, any device driven in the nonlinear regime could present a chaotic regime, extending the range of applications of this complex phenomenon.

In the acoustic community, chaos is at the core of a broad range of discoveries and applications. In liquid, chaos theory explains the complex behavior of strongly driven cavitation bubbles [17]. In infrared imaging, chaotic waves revealed cracks that standard waves could not probe [18]. In sonars, chaotic pulse position modulation (CPPM) enables to identify the origin of the acoustic signal amongst several transducers [19]. Generating chaotic acoustic waves from a PMUT could provide new applications by 
combining the sensitivity of the chaotic regime with the transduction properties of the microstructure.

In this work, we use a PMUT set in the nonlinear Duffing regime to generate chaotic acoustic waves. We present the correlations between the electrical, mechanical and acoustic properties of the generated signal. We then focus on the chaotic acoustic waves and characterize their propagation and directionality. Because of its rich spectrum, we demonstrate how this chaotic ultrasound can be used to jam a signal emitted by a second, linear PMUT. Due to the large bandwidth of the chaotic signal, the driving frequency of the jamming PMUT does not need to match exactly the resonance frequency of the linear PMUT. This chaotic transducer will give new opportunities and will provide disruptive applications ranging from rangefinder to wireless communication security.

\section{RESULTS}

\section{Chaos generation setup}

The PMUT we use consists of a silicon microdiaphragm with a radius of $750 \mu \mathrm{m}$ and a thickness of $10 \mu \mathrm{m}$, fabricated with the PiezoMUMPS process of the Memscap company. A similar device using the same process and geometry was previously reported, presenting interesting properties for rangefinder applications [20]. The device can be seen as a two-port network with the inner and outer electrodes of the AlN piezoelectric layer enabling its actuation and detection (Fig. 1, inset), performed with an Agilent InfiniiVision oscilloscope and a Zurich Instrument HF2LI lock-in amplifier (LIA) assisted by a HF2TA current amplifier. In addition to the piezoelectric transduction scheme, we performed mechanical measurements of the structure velocity with a laser-based Polytech velocimeter OFV3001 and an acoustic detection of the ultrasonic waves generated by the PMUT with a Brüel \& Kjaer 4939-A measuring microphone. All measurements are performed in air, and all amplitudes present in the paper are in root-mean-square.

The mechanical dynamics of the structure follows at first order the canonical equation:

$$
\ddot{x}+\Delta \omega \dot{x}+\omega_{0}^{2} x+\frac{8 \omega_{0}}{3} \alpha x^{3}=\frac{F(t)}{m},
$$

with $x$ the displacement of the membrane, $m$ its mass, $\omega_{0}=2 \pi \times 71.3 \mathrm{kHz}$ the angular resonance frequency and $\Delta \omega=2 \pi \times 750 \mathrm{~Hz}$ the angular bandwidth of the resonator, leading to the quality factor of 95 (Fig. 1). As the amplitude $F_{0}$ of the driving force $F=F_{0} \cos \omega t$ increases (in our case, resulting from a voltage applied on the piezoelectric layer of the device), the structure enters in the Duffing regime. This regime is mainly known to induce a shift in the resonance frequency proportional to the square of the amplitude of the vibrating MEMS [21], [22], and we measure in our case a nonlinear Duffing coefficient of $\alpha=$ $2 \pi \times 54.5 \mathrm{kHz} / \mathrm{V}^{2}$. In this Duffing regime, the resonator presents a hysteresis with two available states, vibrating either at low or at high amplitude depending on its history (Fig. 1, blue line). As the driving angular frequency $\omega$ reaches one of the edges of the hysteresis, the system bifurcates from one state to the other one [23]. This switching is at the core of the chaotic regime exploited in this paper, already introduced as a dynamical bistability based chaos [16].

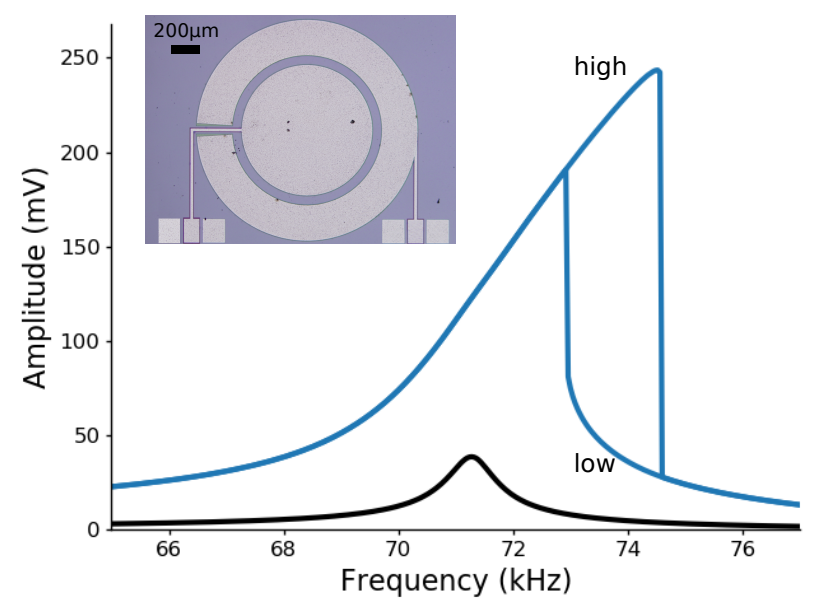

Figure 1: Frequency sweep of the PMUT in the linear (black line, $0.5 \mathrm{~V}$ ) and in the Duffing regimes (blue line, $5 \mathrm{~V}$ ), highlighting the frequency shift and bistability of the nonlinear regime compared to the linear resonance. Inset: microphotography of the PMUT.

By modulating the frequency of the driving force with $F=F_{0} \cos [\omega t+\sin (\delta \omega t)]$ over a large enough range within the hysteresis, the resonator periodically switches between the two states, following:

$$
\begin{gathered}
\dot{R}=-\frac{\Delta \omega}{2} R-\frac{F}{2 m \omega} \sin \varphi, \\
\dot{\varphi}=\omega_{0}-\omega-\delta \omega \cos (\delta \omega t)+\alpha|R|^{2}-\frac{F}{2 m \omega R} \cos \varphi,
\end{gathered}
$$

with $R$ the amplitude of the vibration, $\varphi$ its phase delay with the driving signal, $\omega$ the driving angular frequency and $\delta \omega$ the angular modulation frequency.

As the modulation rate increases, the system leaves the adiabatic regime where the two states represent stable amplitudes, to enter in a chaotic regime where the state of the resonator oscillates erratically between the high and low branches of the hysteresis. To enter in this exotic state, the driving parameters should be chosen according to the resonator Duffing nonlinearity, first with the appropriate driving amplitude to open the hysteresis, and then with the appropriate frequency driving and modulation in order to switch between the two available states [16].

\section{Electrical characterization}

Considering the different parameters of the nonlinear micromechanical resonator, we drive the PMUT by applying $5 \mathrm{~V}$ at $73.6 \mathrm{kHz}$ (within the hysteresis) on the outer electrode and we perform a frequency modulation of $\delta \omega=2 \pi \times 2 \mathrm{kHz}$ throughout the paper. As a result, the structure vibrates at the modulated frequency with an amplitude that is modulated by a chaotic pattern (Fig. 2). To characterize the chaotic behavior of the system, we focus on the demodulated signal from this point forward, obtained with the LIA by probing the inner electrode of the 
PMUT. Note that electrically speaking, the two-port network is nearly symmetric, such that switching drive and probe connections almost leads to the same results.

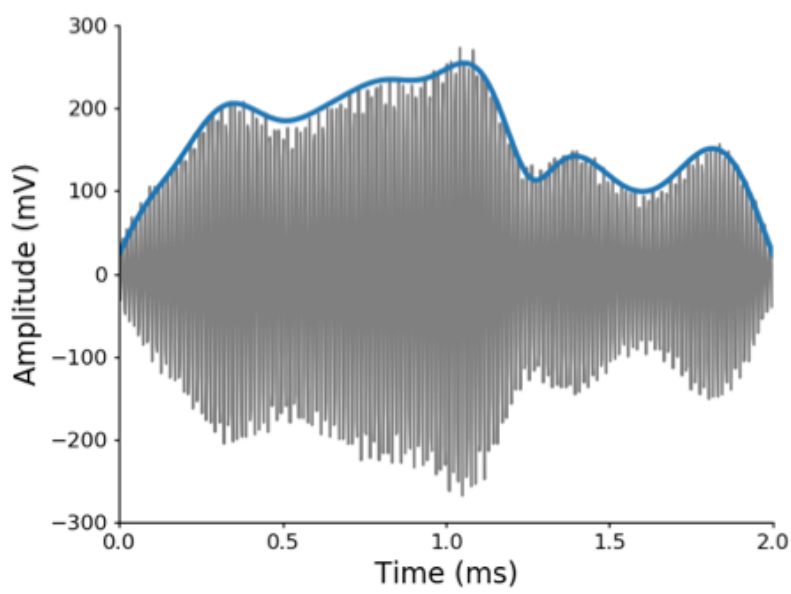

Figure 2: Raw (gray line, output of the oscilloscope) and demodulated (blue line, output of the LIA) measurements of the generated signal in the chaotic regime, representing respectively the displacement $x$ described in (1) and the amplitude $R$ described in (2). This experimental measurement highlights the presence of the chaotic pattern within the envelop of the signal.

One of the most important property of a chaotic system is its sensitivity to the initial conditions. To demonstrate that the PMUT is in a chaotic regime, we initialized the PMUT three times in a row with very similar initial conditions (amplitude, frequency, phase) before switching on the frequency modulation (Fig. 3). All three measurement initially present very similar complex behaviors until they diverge from one another.

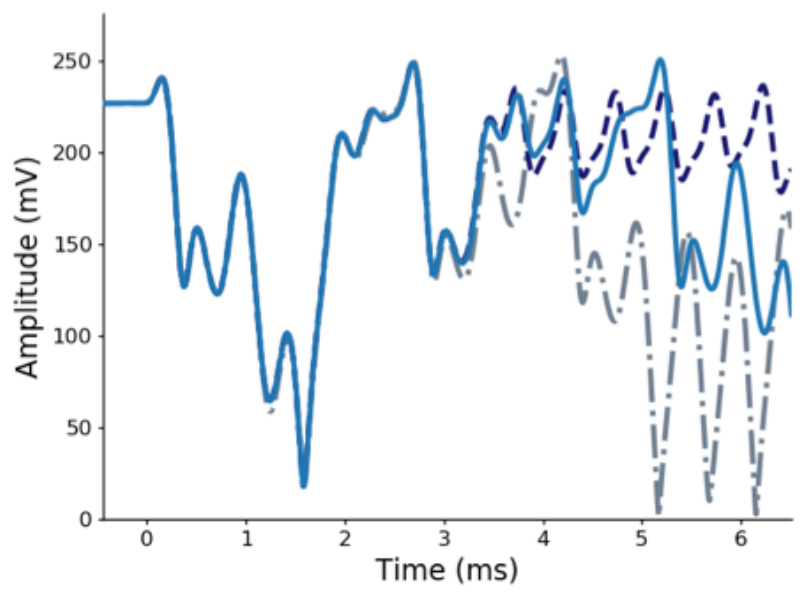

Figure 3: Three successive measurements of the PMUT after demodulation. At $t=0 \mathrm{~s}$, the frequency modulation is turned on to trigger the chaotic behavior of the PMUT. Each set of data was acquired using similar initial conditions just before the modulation.

These different behaviors, at the core of the chaos theory, are the result of the small deviations in the initial conditions that exponentially spread over time, until reaching the size of the signal itself. In this example, the signal amplitude is drastically different after $4 \mathrm{~ms}$ in the chaotic regime.

\section{Mechanical characterization}

The acoustic waves generated by a PMUT result from the displacement of the mechanical structure. While the piezoelectric layer of the MEMS provides information on the device displacement, the complexity of the chaotic regime and its non-periodicity require to characterize how accurate the piezoelectric signal reflects indeed the structure chaotic dynamics. We therefore compared the electrical signal obtained from the piezoelectric layer to that of the velocimeter, measured simultaneously for $100 \mathrm{~ms}$, to demonstrate that the piezoelectric coupling provides most of the information of the microdiaphragm vibration (partially shown in Fig. 4).
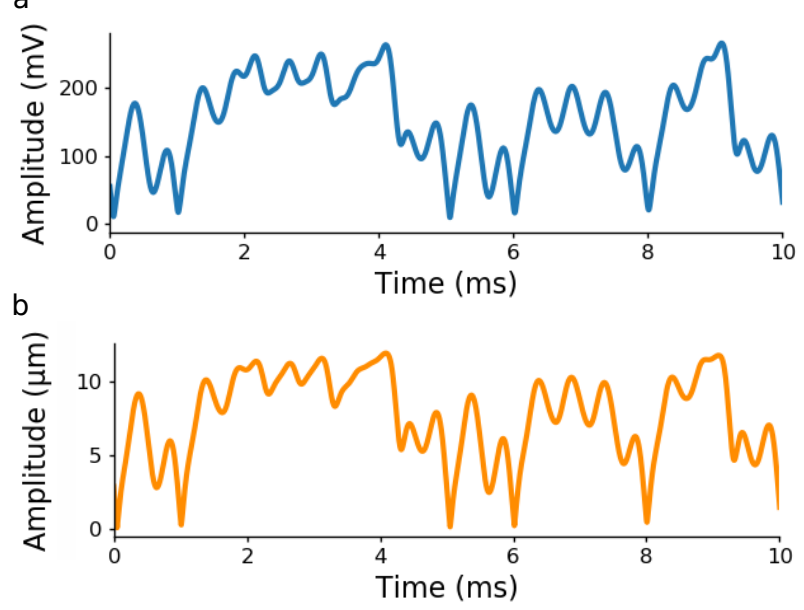

Figure 4: a) Electrical measurement of the chaotic signal; b) Velocimeter output of the same signal, demonstrating high fidelity between piezoelectric and mechanical measurements.

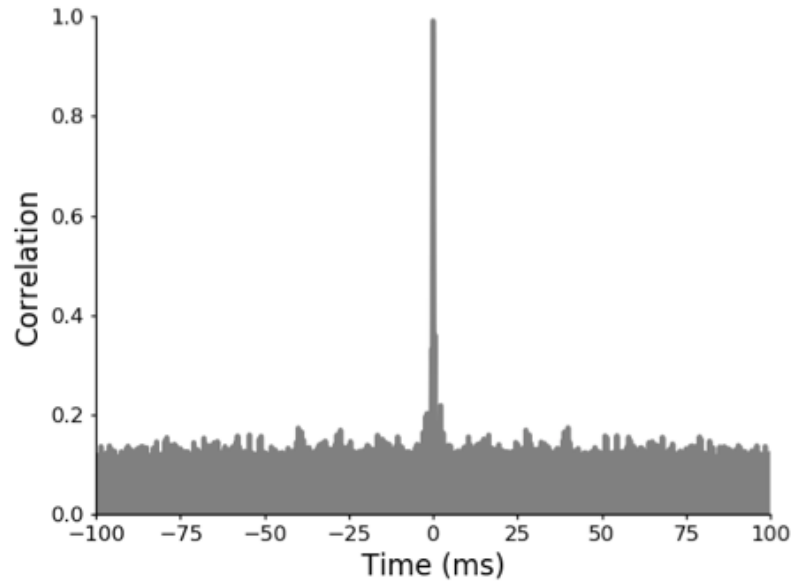

Figure 5: Absolute value of the correlation function between the electrical and mechanical measurements. Because of the non-periodicity of chaos, correlation only exists at $t=0 \mathrm{~s}$, where it reaches more than $99 \%$.

Note that the displacement needed to obtain such chaotic regime is of the order of the thickness of the microdiaphragm. The correlation between the two 
measurements is above $99 \%$ at $\mathrm{t}=0 \mathrm{~s}$ (Fig. 5), demonstrating that the piezoelectric detection reflects the structure dynamics. The lack of correlation elsewhere is another demonstration of the non-periodicity of the chaotic regime, at the core of the CPPM technique for transducer identification [19].

\section{Acoustic characterization}

To characterize the ultrasounds generated by the PMUT, we placed a microphone in front of the microdiaphragm to probe the acoustic signals emitted, which we compare with the electrical measurements of the structure's vibration. Special care was taken to avoid acoustic reverberation using foam to damp the acoustic waves reflected by the equipment. We demonstrate that the chaotic modulation propagates in the air with a waveform similar to the that of electrical measurement (Fig. 6). The delay of the microphone measurement arises from the TOF of the acoustic wave from the device to the microphone,

a

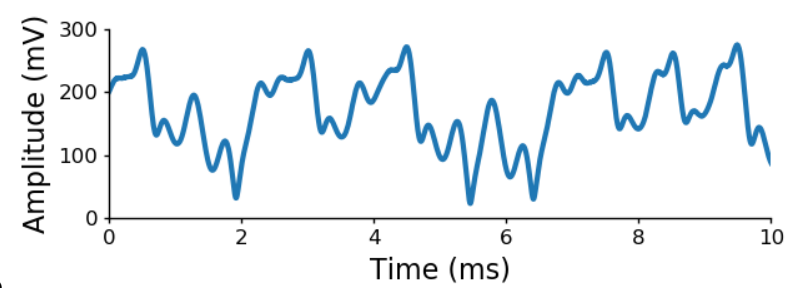

b

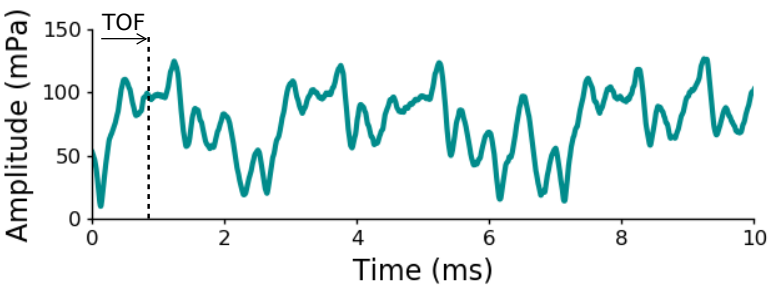

Figure 6: a) Electrical measurement of the chaotic signal; b) Acoustic measurement of the same signal. Because the microphone is $25 \mathrm{~cm}$ away from the PMUT, there is a delay due to the TOF of $0.73 \mathrm{~ms}$.

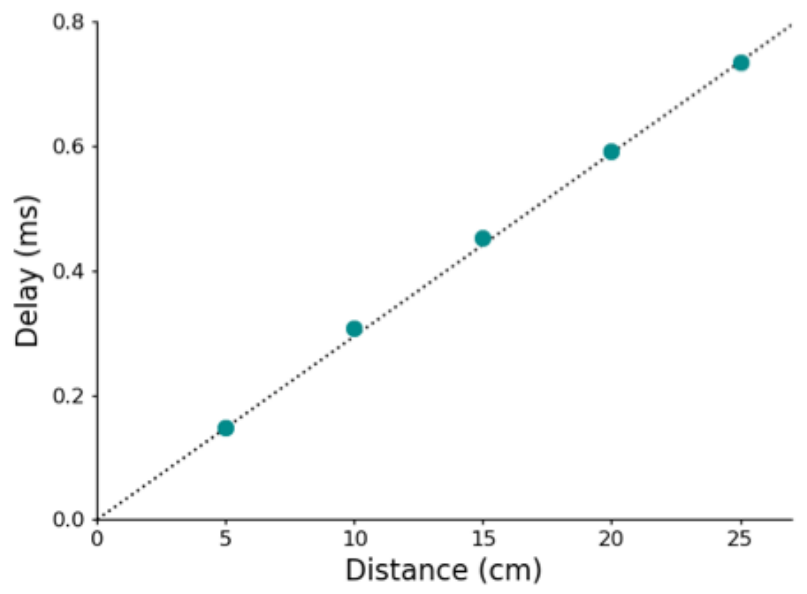

Figure 7: Comparison between the delay of the microphone and the distance to the PMUT. The dotted line corresponds to the speed of sound. which is consistent with the speed of sound in air at room temperature (Fig. 7). While the delayed waveform is mostly the same from the piezoelectric (and therefore mechanical) to the acoustic domains, some features in Fig. 6 a are not reproduced in Fig. 6 b, which is attributed to residual acoustic reflections altering the measurement.

To exploit the complex behavior of the chaotic wave, understanding its directionality is of paramount importance. We characterized the acoustic beam profile at $25 \mathrm{~cm}$ from the PMUT in the linear regime, presenting expected lobes but slightly distorted, which we attribute to the non-anechoic experimental setup and the nonoptimized packaging (Fig. 8 a). We compared this beam profile with the one measured in the chaotic regime. The overall shape presents similar features, revealing that the chaotic modulation does not alter the acoustic propagation of the signal, even for a non-ideal beam profile.

Chaotic signals can be associated to noise, and its dynamics possesses unique properties with applications in cryptography [24] or weak signals detection [25]. In acoustics, chaos enables complex and reliable communication schemes [19]. The integrity of the chaotic waveform as it propagates is therefore essential to preserve its properties for further applications. Remarkably, despite the anisotropic beam profile of the PMUT, the correlation between the electrical and acoustic measurements remains close to 1 in all directions (Fig. 8 b). In average, the measured correlation is above 95\%. For comparison, distinct acoustical chaotic signals generated by the PMUT lead in average to a correlation smaller than $5 \%$.

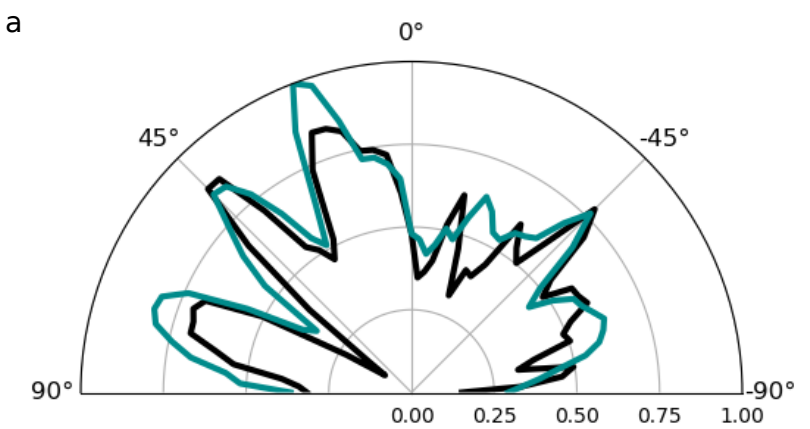

Amplitude profile (norm.)

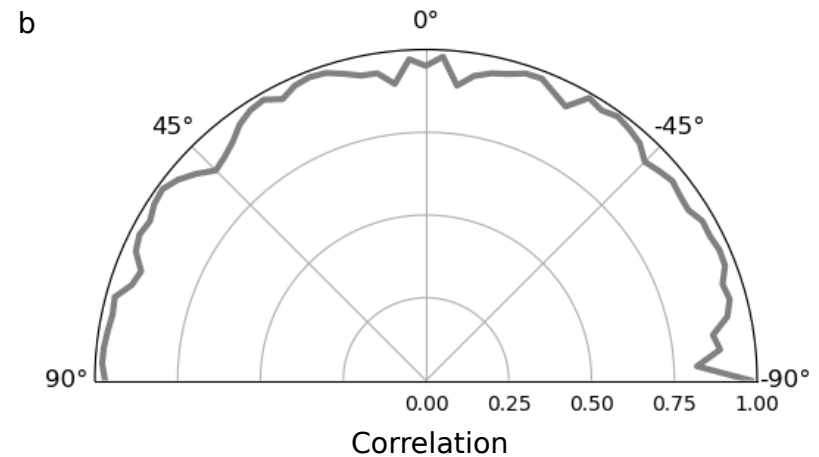

Figure 8: a) Acoustic beam profile of the PMUT in the linear (black line) and chaotic (dark cyan line) regimes, $0^{\circ}$ corresponding to a perfect alignment between the PMUT and the microphone; b) Correlation between the acoustic and piezoelectric signals as a function of directionality. 


\section{Ultrasonic jammer}

One of the primary use of a PMUT is as a rangefinder, using the echolocation principle known from bat's sonar [26]. This technique is the primary hunting tool of this predator, such that their preys learned to counteract their sonar by generating interfering ultrasonic waves themselves [27]. In addition, bats jam other bats using frequency modulated signals during food competition [28]. In a similar fashion, the broad frequency spectrum of the chaotic signal generated by a PMUT could be used to jam signals emitted by other ultrasound transducers.

To demonstrate the jamming potential of PMUT based chaotic ultrasounds, we will use two similar PMUT devices, one working in the chaotic and the other in the linear regime. The chaotic PMUT device, excited by a harmonic signal of $5 \mathrm{~V}$ at $73.6 \mathrm{kHz}$ with a frequency modulation of $2 \mathrm{kHz}$, is placed close to the other PMUT driven in the linear regime at its resonance frequency of $71.3 \mathrm{kHz}$ by a sinusoidal signal of $0.5 \mathrm{~V}$. Note that both driving frequencies are more than three PMUT bandwidths away. When both PMUTs are simultaneously driven, pointing towards the measuring microphone, the linear PMUT signal is mixed with the one of the chaotic PMUT, which may result in a total loss of information.

In a standard rangefinder operation, the PMUT is alternatively used as an actuator and as a sensor, generating and receiving the ultrasonic waves. As an actuator, it is turned on by applying a short burst at the resonance frequency, which length is the result of a compromise to probe both long and short distances. The minimum number of cycles to obtain large enough acoustic waves is traditionally given by the quality factor of the resonator, leading to a burst time length of $t_{b}=\frac{2 \pi}{\Delta \omega}$, also corresponding to the PMUT acquisition time as a sensor.

For the jammer proof-of-concept, we drove the PMUTs with a continuous signal instead of a burst, but using a limited acquisition time to mimic the sensing phase of rangefinders. If we use an acquisition time of $t_{b}$ (in our case, around $1 \mathrm{~ms}$ ) in the presence of the chaotic PMUT, all the information of the harmonic wave is lost within the broadband chaotic signal (Fig. 9 a). Such short acquisition times lead to poorly resolved Fourier transforms, on top of which the noisy structure of the chaotic ultrasound drowns the linear signal. However, with a longer acquisition time, this noise-like pattern can be averaged down to recover the ultrasound of the linear PMUT (Fig. 9 b). To demonstrate this, we analyze the fast Fourier transform of the output of the microphone at the driving frequency of the linear PMUT $(71.3 \mathrm{kHz})$ as a function of the acquisition time. This study is done with two sets of measurement: when the chaotic PMUT alone is driven and when it is combined with the linear PMUT. Each measurement was performed one hundred times to obtain enough statistics. We then define that the linear acoustic signal can be unambiguously detected if the amplitude difference between the two sets is larger than three standard deviations. We observe that the harmonic signal emerges back after an acquisition time of $16 t_{b}$ (Fig. 10). This longer acquisition time could have disastrous consequences, as it blinds the PMUT rangefinder for a substantial amount of time. This jamming application is directly related to the unique properties of chaos, because it is a non-periodic and an unpredictable signal that cannot simply be filtered or subtracted. Note that reducing the burst time implies a lower acoustic wave amplitude, being even more difficult to probe, and therefore does not bypass this problem.

a
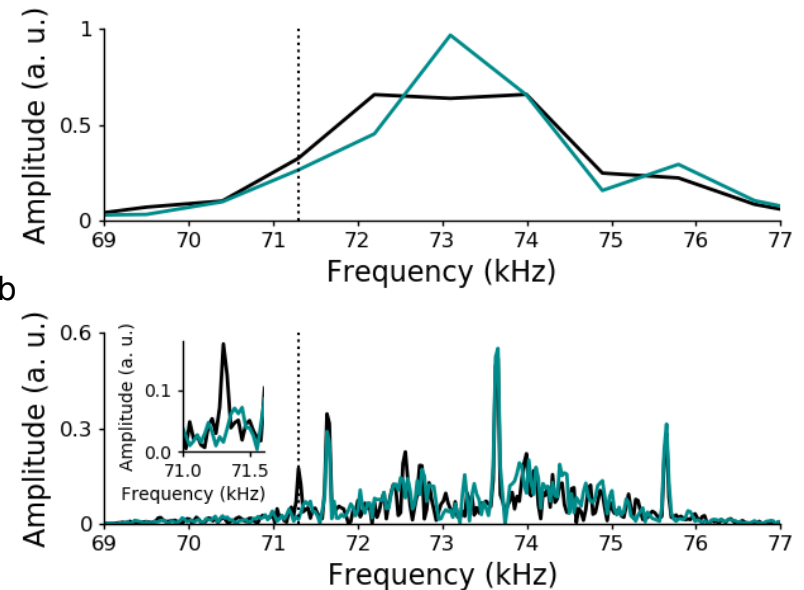

Figure 9: a) Acoustic frequency spectrum of the chaotic PMUT alone (dark cyan) and combined with the linear PMUT (black) vibrating at $71.3 \mathrm{kHz}$ (black dashed line), after a standard acquisition time of $t_{b} \approx 1 \mathrm{~ms}$. This low acquisition time leads to a poorly resolved Fourier transform, where the harmonic signal is completely drowned out by the broad spectrum of the chaotic ultrasound. b) Similar frequency spectrum after a longer acquisition time of $32 t_{b}$. The higher frequency resolution enables to see the career signal of the chaotic PMUT at $73.6 \mathrm{kHz}$ as well as satellites peaks at $\pm 2 \mathrm{kHz}$ corresponding to the frequency modulation. The inset zoom in the frequency range of the linear PMUT, which peak is now clearly distinguishable from the chaotic signal.

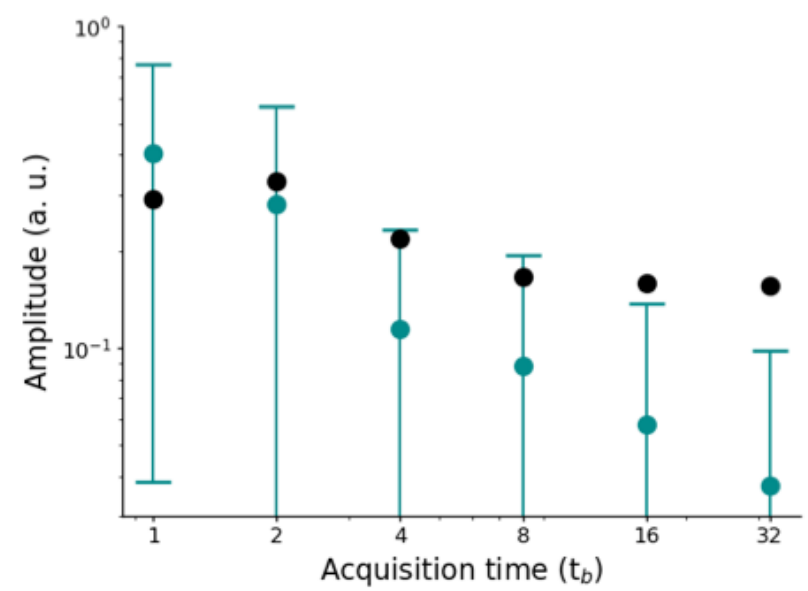

Figure 10: Amplitude of the FFT at $71.3 \mathrm{kHz}$ of the chaotic PMUT alone (dark cyan) and combined with the linear PMUT (black) for different acquisition times, averaged over 100 measurements. The error bars represent the three standard deviations of the chaotic PMUT, above which the linear signal can be considered as recovered.

This jamming application only requires for the PMUT to be driven in the nonlinear regime with a 
frequency modulation configuration, where the broad frequency spectrum directly arise from the nonlinear dynamics of the structure. It is therefore directly applicable to any PMUT, and more generally to any acoustic transducer, as long as its Duffing regime is accessible. By carefully selecting the appropriate set of parameters, this chaotic regime could spread over a wider spectrum with a higher amplitude, enabling to cover stronger signals on a large frequency range.

\section{CONCLUSION}

This work presents the generation and characterization of a chaos modulated ultrasound wave. This acoustic signal was generated by a PMUT set in the Duffing regime, which driving frequency was modulated to enter in the chaotic regime. The propagation of the chaotic waves follows the properties of the linear PMUT and the specific pattern of the chaotic modulation remains unaltered in all directions. We finally demonstrate that the rich spectrum of the chaotic signal can be used as a jammer to cover nearby ultrasonic waves.

Beside its noisy-like structure, chaotic signals demonstrated to have unique properties, in particular for secured communication [12]. The implementation of chaos in a physical microtransducer described in this paper brings new opportunities to develop wireless, acoustic-based cryptography and tackle the long-standing challenge of security in IoT technologies.

\section{ACKNOWLEDGEMENTS}

The authors acknowledge financial support from the INS2I - CNRS grant under the project BatCipher. The authors thank Gaëtan Debontride for insightful discussions regarding the instrumentation techniques.

\section{CONTACT}

\section{*martial.defoort@univ-grenoble-alpes.fr}

\section{REFERENCES}

[1] J. Dong, X. Li, Y. Wang, D. Lu, and S. Ahat, 'Silicon micromachined high-shock accelerometers with a curvedsurface-application structure for over-range stop protection and free-mode-resonance depression', J. Micromech. Microeng., vol. 12, no. 6, p. 742, 2002.

[2] C. H. Ahn et al., 'Geometric compensation of (100) single crystal silicon disk resonating gyroscope for modematching', in 2013 Transducers Eurosensors XXVII: The 17th International Conference on Solid-State Sensors, Actuators and Microsystems (TRANSDUCERS EUROSENSORS XXVII), 2013.

[3] J. W. Weigold, T. J. Brosnihan, J. Bergeron, and X. Zhang, 'A MEMS Condenser Microphone for Consumer Applications', in 19th IEEE International Conference on Micro Electro Mechanical Systems, 2006.

[4] G. M. Rebeiz and J. B. Muldavin, 'RF MEMS switches and switch circuits', IEEE Microwave Magazine, vol. 2, no. 4, pp. 59-71, 2001.

[5] R. Przybyla et al., 'An ultrasonic rangefinder based on an AlN piezoelectric micromachined ultrasound transducer', in 2010 IEEE SENSORS, 2010.

[6] B. Herrera, F. Pop, C. Cassella, and M. Rinaldi, 'AlN PMUT-based Ultrasonic Power Transfer Links for Implantable Electronics', in 2019 20th International Conference on Solid-State Sensors, Actuators and Microsystems Eurosensors XXXIII (TRANSDUCERS EUROSENSORS XXXIII), 2019.

[7] X. Liu, D. Chen, D. Yang, X. Chen, X. Le, and J. Xie, 'A Computational Piezoelectric Micro-Machined Ultrasonic Transducer Toward Acoustic Communication', IEEE Electron Device Letters, vol. 40, no. 6, pp. 965-968, 2019.

[8] R. Almog, S. Zaitsev, O. Shtempluck, and E. Buks, 'Signal amplification in a nanomechanical Duffing resonator via stochastic resonance', Applied Physics Letters, vol. 90, no. 1, p. 013508, 2007.

[9] M. Defoort, P. Taheri-Tehrani, and D. A. Horsley, 'Exploiting nonlinear amplitude-frequency dependence for temperature compensation in silicon micromechanical resonators', Appl. Phys. Lett., vol. 109, no. 15, p. 153502, 2016.

[10] T. L. Carroll, 'A simple circuit for demonstrating regular and synchronized chaos', American Journal of Physics, vol. 63, no. 4, pp. 377-379, 1995.

[11] T. Stojanovski and L. Kocarev, 'Chaos-based random number generators-part I: analysis [cryptography]', IEEE Transactions on Circuits and Systems I: Fundamental Theory and Applications, vol. 48, no. 3, pp. 281-288, 2001.

[12] A. Argyris et al., 'Chaos-based communications at high bit rates using commercial fibre-optic links', Nature, vol. 438, no. 7066, pp. 343-346, 2005.

[13] Y. C. Wang, S. G. Adams, J. S. Thorp, N. C. MacDonald, $P$. Hartwell, and F. Bertsch, 'Chaos in MEMS, parameter estimation and its potential application', IEEE Transactions on Circuits and Systems I: Fundamental Theory and Applications, vol. 45, no. 10, pp. 1013-1020, 1998.

[14] S. Houri, M. Asano, H. Yamaguchi, N. Yoshimura, Y. Koike, and L. Minati, 'Generic Rotating-Frame-Based Approach to Chaos Generation in Nonlinear Micro- and Nanoelectromechanical System Resonators', Phys. Rev. Lett., vol. 125, no. 17, p. 174301, 2020.

[15] G. Madiot, F. Correia, S. Barbay, and R. Braive, 'Bichromatic synchronized chaos in coupled optomechanical nanoresonators', arXiv:2005.08896 [nlin, physics:physics], 2020.

[16] M. Defoort, L. Rufer, L. Fesquet, and S. Basrour, 'A dynamical approach to generate chaos in a micromechanical resonator', arXiv:2101.11976 [nlin, physics:physics], 2021.

[17] W. Lauterborn and J. Holzfuss, 'Acoustic chaos', Int. J. Bifurcation Chaos, vol. 01, no. 01, pp. 13-26, 1991.

[18] X. Han et al., 'Acoustic chaos for enhanced detectability of cracks by sonic infrared imaging', Journal of Applied Physics, vol. 95, no. 7, pp. 3792-3797, 2004.

[19] L. Fortuna, M. Frasca, and A. Rizzo, 'Chaotic pulse position modulation to improve the efficiency of sonar sensors', IEEE Transactions on Instrumentation and Measurement, vol. 52, no. 6, pp. 1809-1814, 2003.

[20] A. Suresh, K. L. Mak, J. Benserhir, J. E.-Y. Lee, and L. Rufer, 'Air-coupled Ultrasonic Rangefinder with Meterlong Detection Range Based on a Dual-electrode PMUT Fabricated Using a Multi-user MEMS Process', in 2019 IEEE SENSORS, 2019.

[21] I. Kozinsky, H. W. Ch. Postma, I. Bargatin, and M. L. Roukes, 'Tuning nonlinearity, dynamic range, and frequency of nanomechanical resonators', Appl. Phys. Lett., vol. 88, no. 25, p. 253101, 2006.

[22] M. Marzencki, M. Defosseux, and S. Basrour, 'MEMS 
Vibration Energy Harvesting Devices With Passive Resonance Frequency Adaptation Capability', Journal of Microelectromechanical Systems, vol. 18, no. 6, pp. 14441453, 2009.

[23] M. Defoort, V. Puller, O. Bourgeois, F. Pistolesi, and E. Collin, 'Scaling laws for the bifurcation escape rate in a nanomechanical resonator', Phys. Rev. E, vol. 92, no. 5, p. 050903, 2015.

[24] L. O. Chua, M. Itoh, L. Kocarev, and K. Eckert, 'Chaos synchronization in chua's circuit', J CIRCUIT SYST COMP, vol. 03, no. 01, pp. 93-108, 1993.

[25] Guanyu Wang, Dajun Chen, Jianya Lin, and Xing Chen, 'The application of chaotic oscillators to weak signal detection', IEEE Transactions on Industrial Electronics, vol. 46, no. 2, pp. 440-444, 1999.

[26] G. Jones and M. W. Holderied, 'Bat echolocation calls: adaptation and convergent evolution', Proceedings of the Royal Society B: Biological Sciences, vol. 274, no. 1612, pp. 905-912, 2007.

[27] A. J. Corcoran, J. R. Barber, and W. E. Conner, 'Tiger Moth Jams Bat Sonar', Science, vol. 325, no. 5938, pp. 325-327, 2009.

[28] A. J. Corcoran and W. E. Conner, 'Bats jamming bats: Food competition through sonar interference', Science, vol. 346, no. 6210, pp. 745-747, 2014. 